تاثير تزريق درون تخممرغى عصاره ترده كاج بر رشد و تمايز جنسيتى جوجههاى توشتى

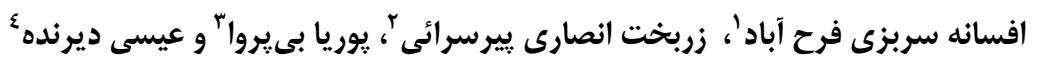

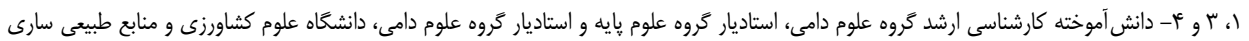

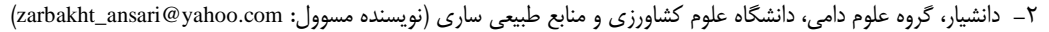

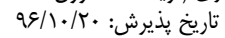
تاريخ دريافت:

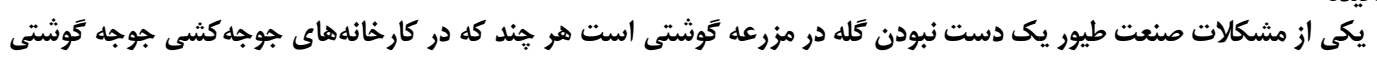

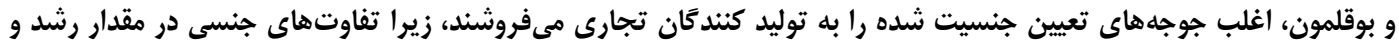

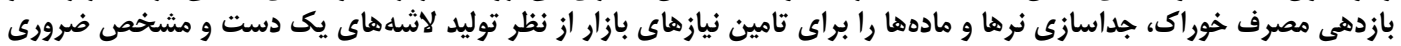

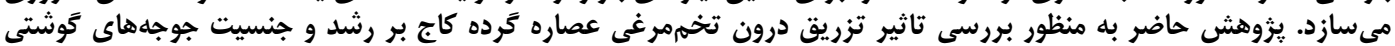

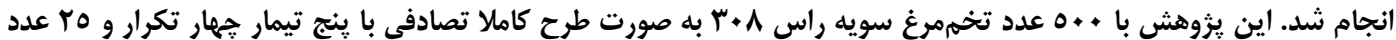

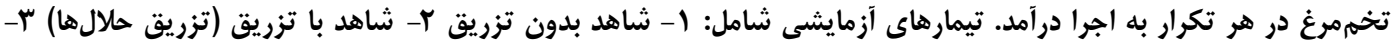

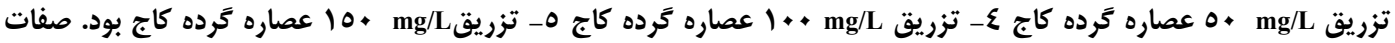

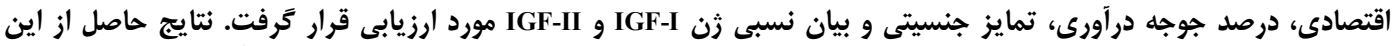

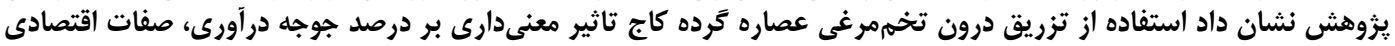

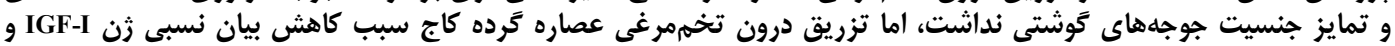
IGF-II

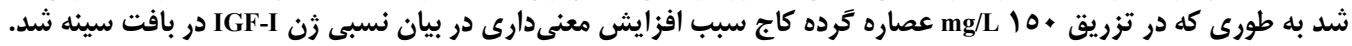

وازهاى كليدى: تزريق درون تخممرغى، تمايز جنسيتى، رشد، عصاره كرده كاج

بازدارنده آروماتاز در مرحلهاى كه گَناد جوجه نامتمايز است،

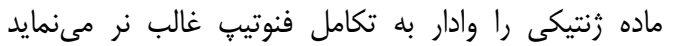

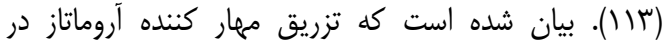

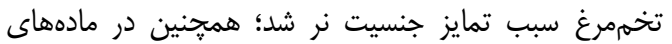

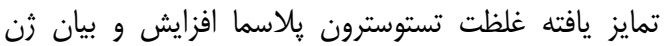

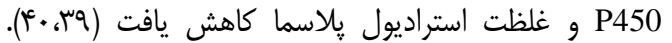

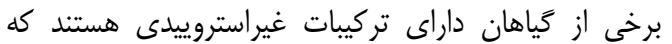

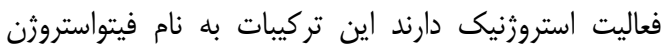

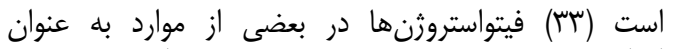

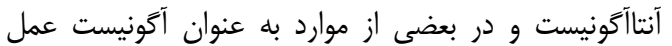

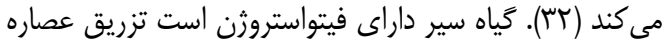

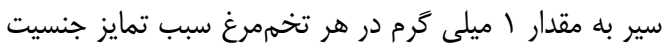

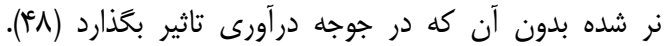

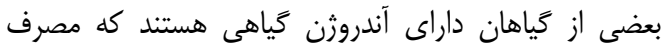

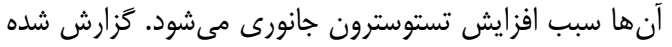

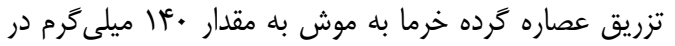

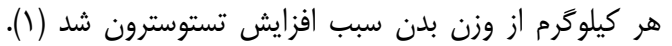

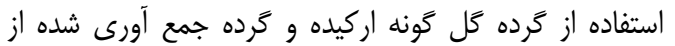

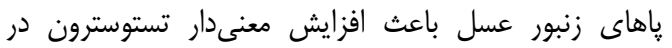

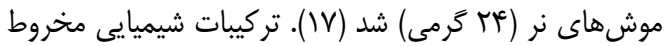

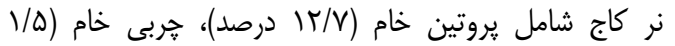

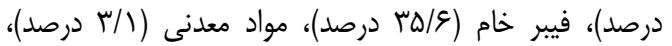

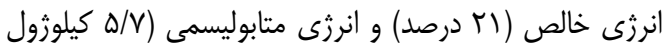

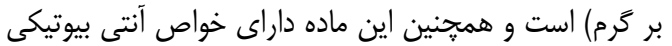

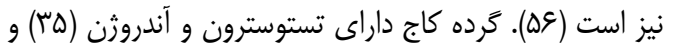

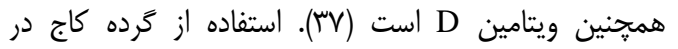

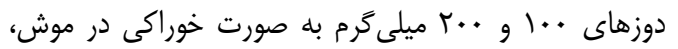

تاريخجه تمايز جنسيت مربوط به قرن نوزدهم است كه

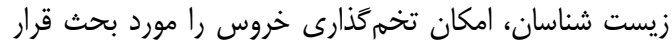

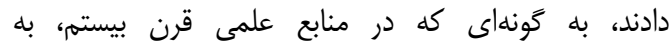

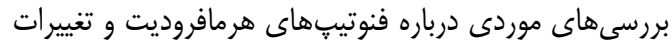

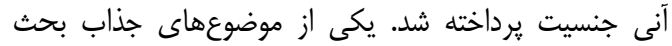

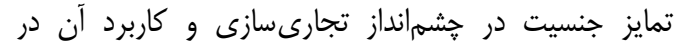

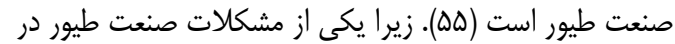

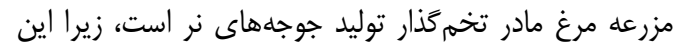

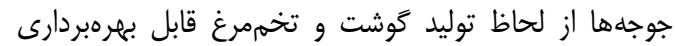

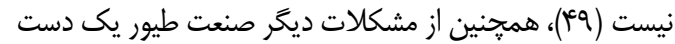

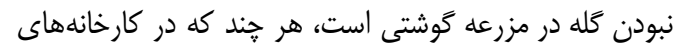

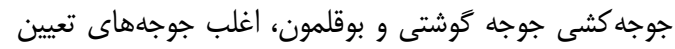

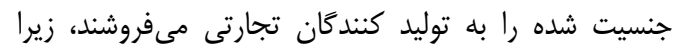

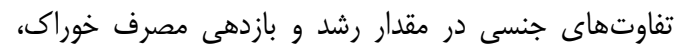

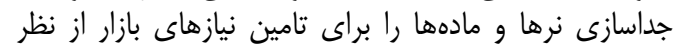

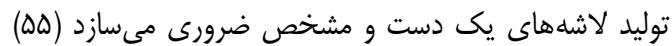

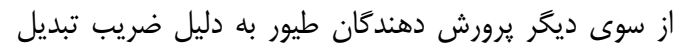

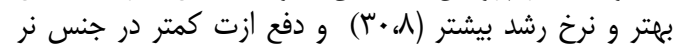

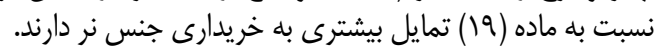

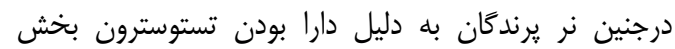

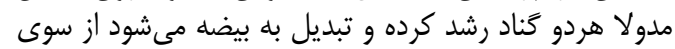

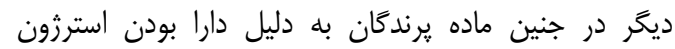

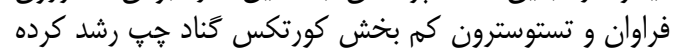

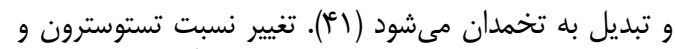

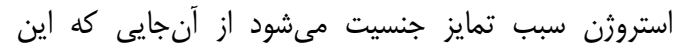

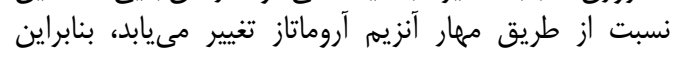


عصاره يك ميلىليتر DMSO به منظور حل حل شدن عصاره

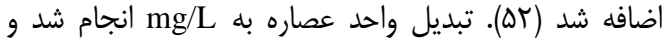
سيس براساس فرمول ميزان دوز مورد نظر با بالون زوزه به براس حجم رسانده شد.

تعيين دوز تيمارها

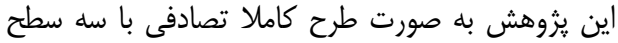

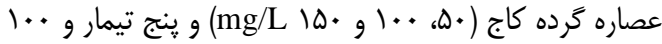

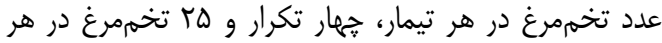

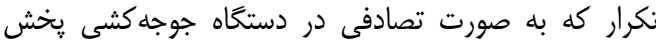

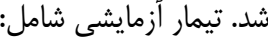

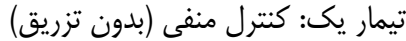

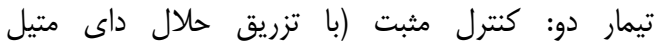

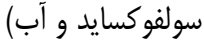

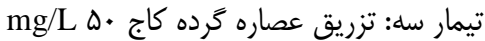

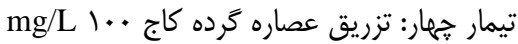

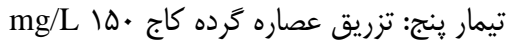

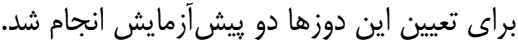
تزريق داخل تخممرغ ترغ

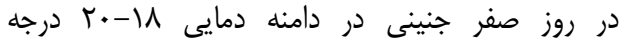

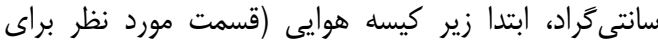

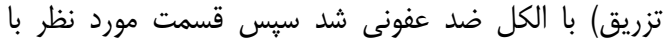

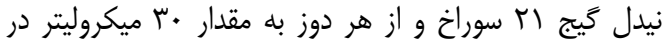

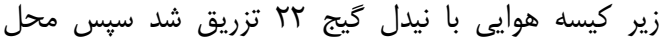

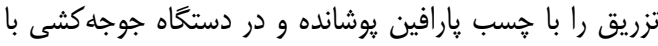

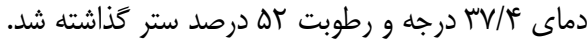

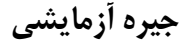

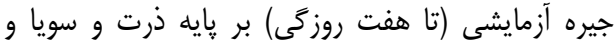

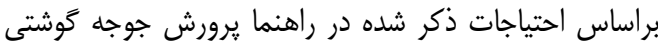

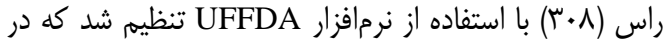

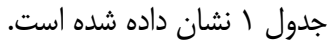

سبب بهبود آرتريت و كاهش التهاب شده و همحِنين سطح

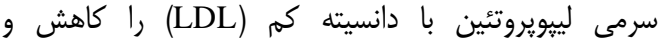

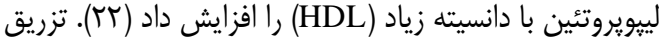

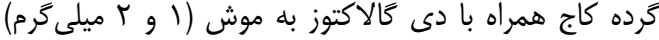

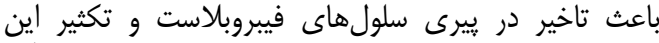

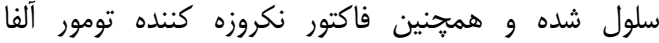
(TNF- $\alpha)$

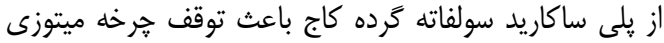

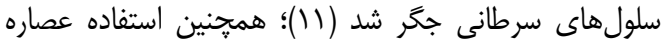

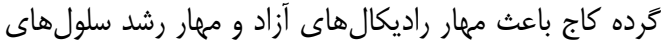
سرطانى شد (Trاج (T). كرده كاج با دارا بودن هورمونهاى فيتواستروثن و واني

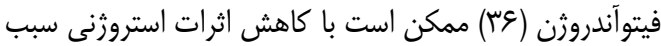

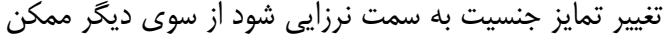

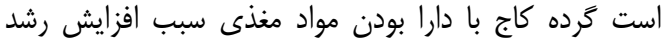

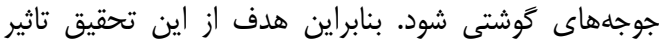

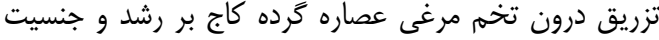
جوجههاى كَشتى بود.

\section{مواد و روشها جمع آورى مخروط كاج}

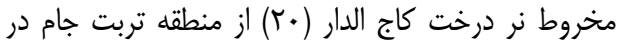

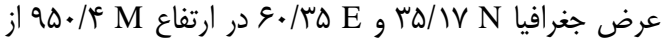
درختان اين شهرستان جمع آورى شدافيا

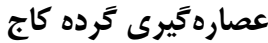

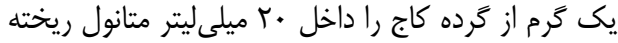

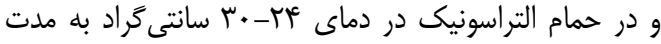

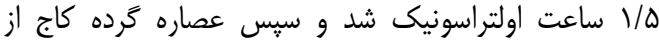

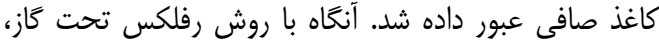

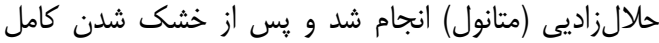

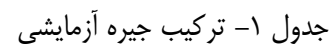

\begin{tabular}{|c|c|}
\hline اجزاى خوراك (درصد) & مواد خوراكى \\
\hline $0 \cdot / 9 \mu$ & ذرت \\
\hline$F \cdot 10 V$ & كنجاله سويا \\
\hline$r / 91$ & روغن \\
\hline I/Va & دى كلسيم \\
\hline $1 / r$ & سنحَ آهى \\
\hline q & نمك \\
\hline.$/ T \Delta$ & مكمل معدنى \\
\hline$\cdot / T \Delta$ & مكمل ويتامينى \\
\hline$\cdot / 4 \wedge$ & دى-ال-متيونين \\
\hline . AT & ليزين \\
\hline.$/ 4 t$ & ترئونين \\
\hline & ادامه جدول.. \\
\hline & تركيب مواد مغذى برحسب درصد \\
\hline rq1. & انرزى (كيلو كالرى /كيلوكرم) \\
\hline$r / \mu$ & يروتين \\
\hline $1 / F^{4}$ & ليزين \\
\hline.$/ 69$ & متيونين \\
\hline $1 / \cdot 1$ & متيونين + سيستئين \\
\hline - /9V & ترئونين \\
\hline$\cdot / 90$ & كلسيم \\
\hline.$/ F V \Delta$ & فسفر \\
\hline.$/ 11$ & سديم \\
\hline
\end{tabular}


(كامه پايانى). ويثزى اين كَامهها در جدول بيان شده است

براى تعيين كيفيت جوجههاى هج شده شآيت شاخصهايى

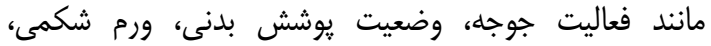

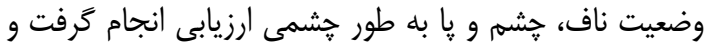

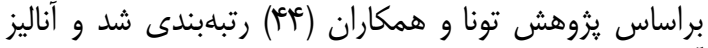

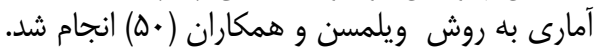

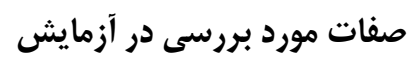

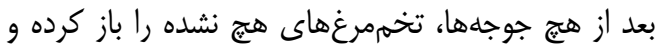

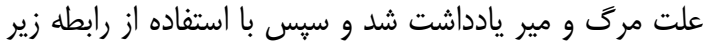
(DI)

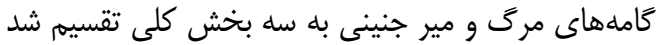

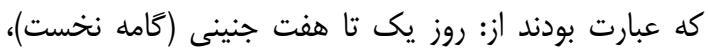

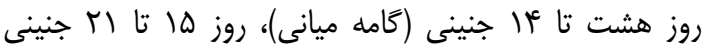

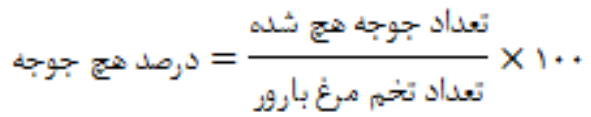

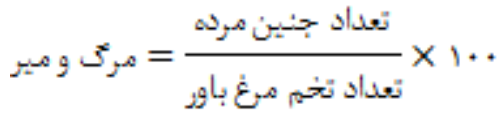

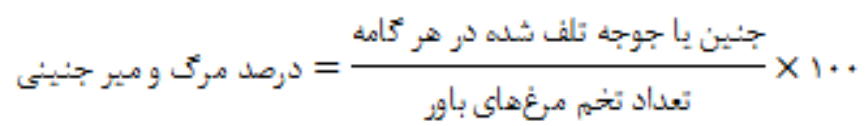

وزن كشى شدند. ضريب تبديل غذايى در دورههاى يزوهش از

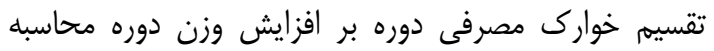
شد. وزن هر واحد در محاسبه ضريب تبديل غذايل دوريى در نظر كرفته شد: شن
براى محاسبه تمايز جنسيت، جوجههاى هج شده را با

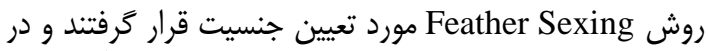

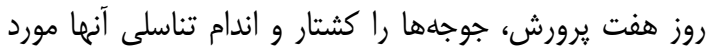

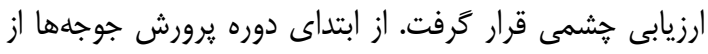

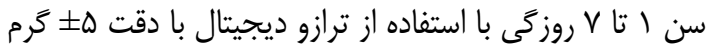

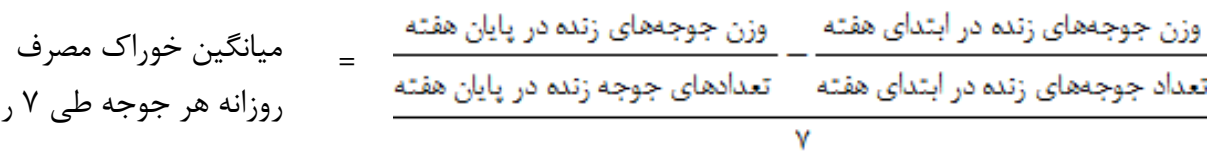

افزايش وزن روزانه هر جوجه= ميانگين افزايش وزن هفتخى هر جوجه XV

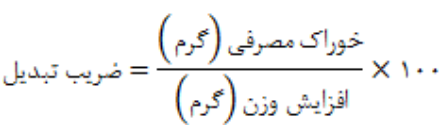

\section{Real-Time PCR نجام إن}

Real Time PCR

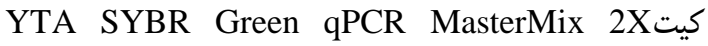

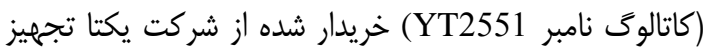

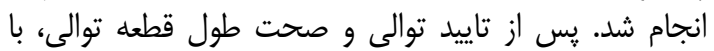

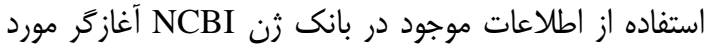

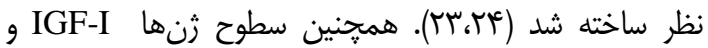

$$
\text { IGFII }
$$

اندازهَيرى بيان خن IGF-I و IGF-II

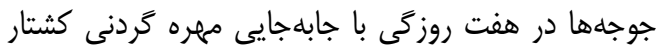

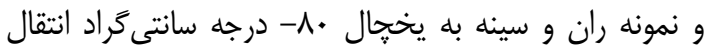
داده شد.

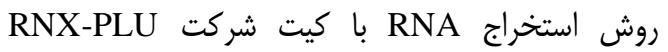

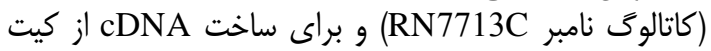
cDNA Synthesis Kit

\begin{tabular}{|c|c|c|c|c|}
\hline $\begin{array}{c}\text { اندازه باند) } \\
\text { (bp) }\end{array}$ & جهت & 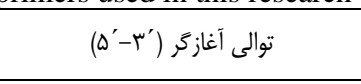 & شماره ثبت در بانك زن & 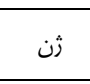 \\
\hline Ime & بركشت & $\begin{array}{l}\text { GAGACAGAGGCTTCTACTTCA } \\
\text { TGGCTTTATTGGAGCACAG }\end{array}$ & NM_001004384.2 & IGF-I \\
\hline 91 & برثشت & $\begin{array}{l}\text { TGTGGAGGAGTGCTGCTTTC } \\
\text { GGGAGGTGGCGGAGAGGTCA }\end{array}$ & NM_001030342.1 & IGF-II \\
\hline r.. & بركشت & $\begin{array}{l}\text { TGCGTGACATCAAGGAGAAG } \\
\text { TGCCAGGGTACATTGTGGTA }\end{array}$ & NM_205518 & $\beta$-Actin \\
\hline
\end{tabular}
YT4500 (استفاده شد.

Table 2. Characteristics of the primers used in this research

Insulin- Like Growth factor-1 :IGF-I ،Insulin-Like Growth factor-2 :IGF-II ، Beta-actin :ßActin 
(Dimethyl sulfoxide)

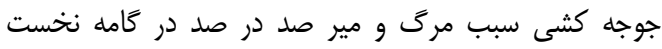

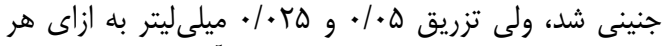

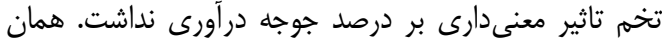

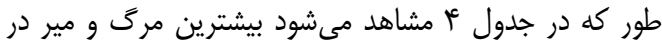

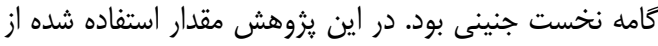

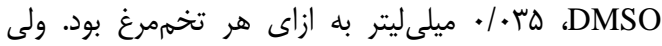

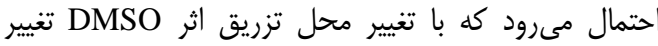

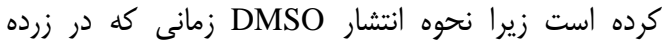

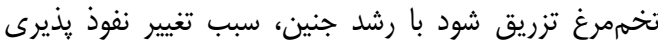

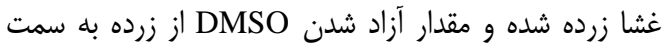

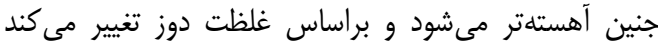

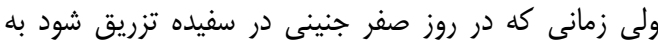

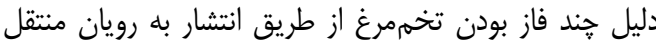

كيفيت جوجله

نتايج مربوط به تاثير تيمارهاى آزمايشى بر كيفيت جوجه

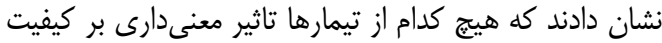

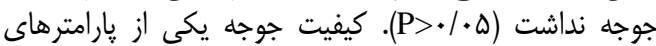

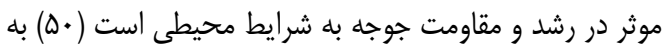

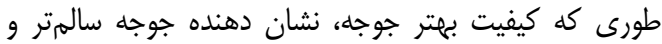

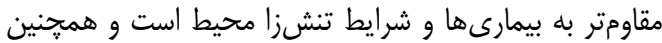
بعد از هج قابليت زنده مانى بيشترى دارد دارد.

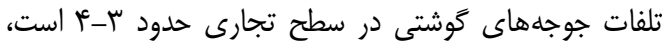

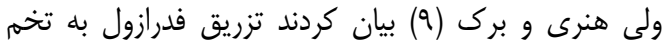

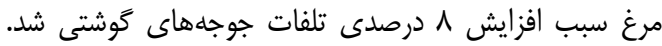

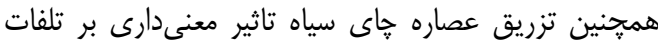

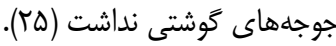

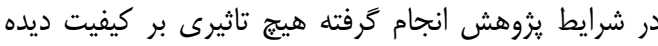

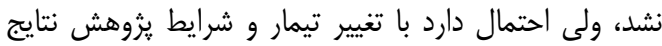

$$
\text { ديگرى به دست آيد. }
$$

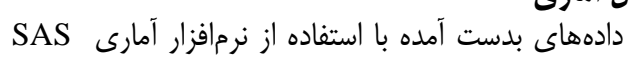

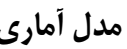

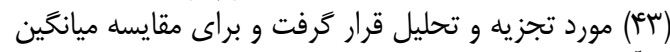
از آزمون هند دامنه دانكن در سطح معنى دارى هـ هـ/. درصد

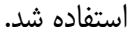

$\mathrm{Y}_{\mathrm{ijk}}=+\mathrm{T}_{\mathrm{i}}+\mathrm{e}_{\mathrm{ijk}}$

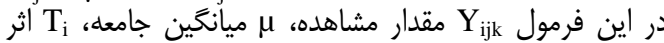

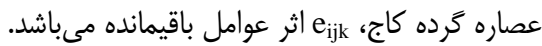

\section{نتايج و بحث}

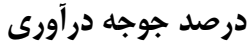

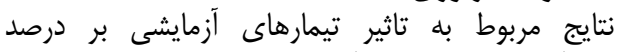
جوجه درآورى در جدول بَ آمده است. بين تيمارهاى ترائ تزريق درايق

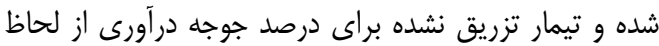

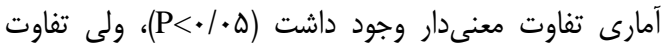

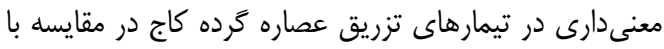

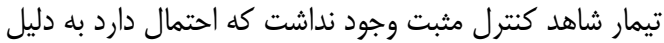

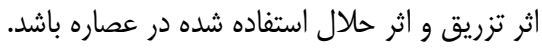

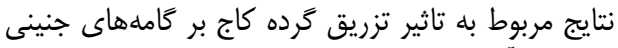

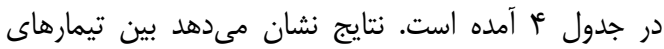

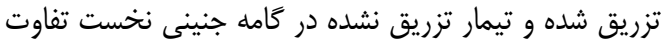

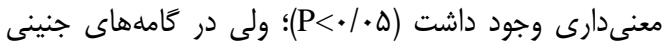
ديخر هيج تفاوت معنى دار مشاهده نشد دارد

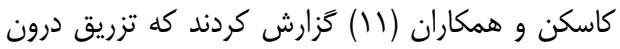

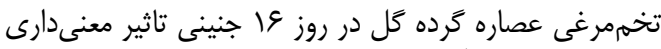

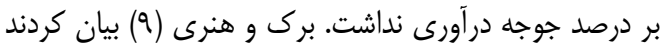

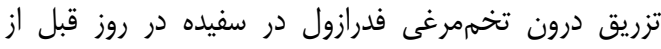

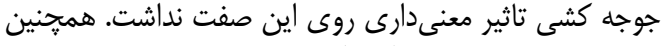

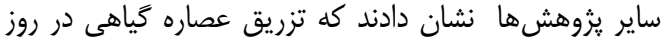

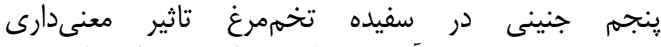

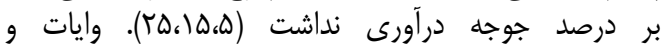

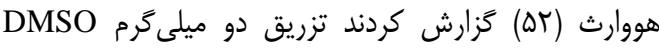

Table 3. Effect of experimental treatments on hatchability and mortality percent

\begin{tabular}{|c|c|c|}
\hline درصد مرك و مير & درصد جوجه درأورى & تيمار \\
\hline$\Lambda^{0}$ & $9 Y / . .^{a}$ & شاهد بدون تزريق \\
\hline$\Delta \backslash / \& Y^{\mathrm{a}}$ & $F \Delta / 9^{D}$ & شاهد با تزريق (DMSO) \\
\hline$\Delta q / 1 .^{a}$ & $T V / \Delta F^{D}$ & (mg/L) ه مصاره گرده كاج (m) \\
\hline$\Delta 1 / \mathrm{F}^{\mathrm{a}}$ & $\uparrow \uparrow / q^{D}$ & عصاره كرده كاج .. (mg/L) \\
\hline$\Delta r / \epsilon V^{a}$ & $\Gamma \Delta / r^{\mathrm{D}}$ & (mg/L)1ه • عصاره كرده كاج \\
\hline 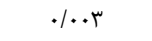 & $\% t$ & P-value \\
\hline$\Delta / \Lambda \mu$ & $9 / . r$ & SEM \\
\hline
\end{tabular}


Table 4. Effect of experimental treatments on embryonic stage percentage

\begin{tabular}{|c|c|c|c|}
\hline "َامه پايانى (روز ها-IIT جنينى) & كامه ميانى (روز ^- أ| جنينى) & كامه نخست (روز (V-1 جنينى) & تيمار \\
\hline $4 /$. &.$/$ & $r^{a}$ & شاهد بدون تزريق \\
\hline $1 / \pi 1$ & $8 / 1 \Delta$ & $(r Y / 9)^{D}$ & شاهد با تزريق (DMSO) \\
\hline ع/r & זr/ץ & $F) / q V^{0}$ & 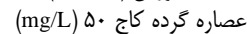 \\
\hline s/qu & $1 / \cdot 1$ & $r \Psi / \Psi^{D}$ & عصاره گرده كاج ..1 (mg/L) \\
\hline$f / \Delta f$ & $t / F T^{2}$ & $r \Delta / v)^{D}$ & عصاره كرده كاج •(mg/L)1D \\
\hline.$/ 4 r$ & זr/. & $\%$ r & P-value \\
\hline سז/. &.$/ 4 V$ & . & SEM \\
\hline
\end{tabular}

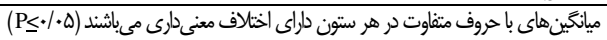

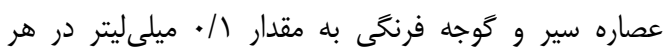

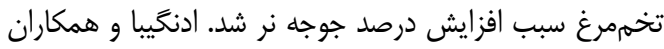

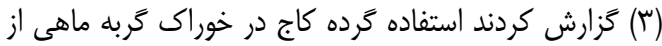

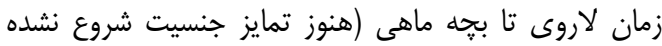

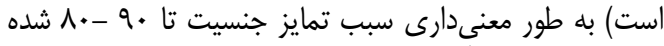

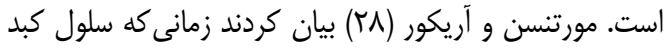

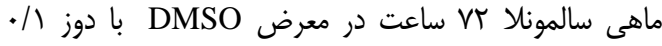

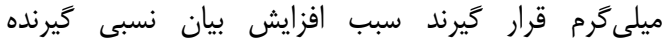

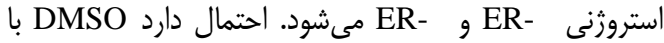
افزايش كيرنده استروثنى عصاره گرده كاج شود.
نتايج مربوط به تيمارهاى آزمايشى و اثر آن بر تمايز

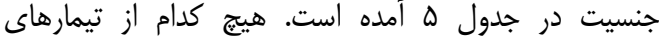

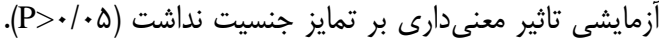

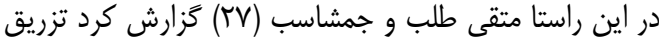

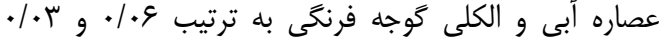

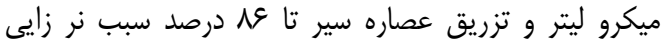

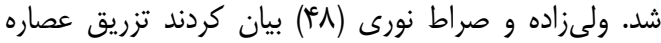
سير به مقدار يك ميلى

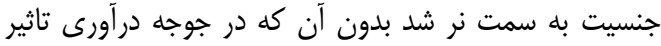

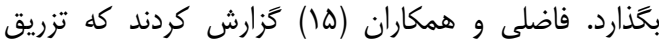

Table 5. Effect of experimental treatments on sex differentiation percentage

جدول ه- تاثير تيمارهاى آزمايشى بر درصد تمايز جنسيت

\begin{tabular}{|c|c|c|}
\hline ماده & ن & تيمار \\
\hline$F \mid / q V$ & $\Delta N / \Gamma$ & شاهد بدون تزريق \\
\hline$\Delta F / Q F$ & $F \Delta / \cdot \Delta$ & شاهد با تزريق (DMSO) \\
\hline TE/ & $\Delta H / \Delta V$ & عصاره كرده كاج • D (mg/L) \\
\hline$r \varphi / T$ & $\Delta \omega / \mathcal{M}$ & عصاره كَرده كاج... (mg/L) \\
\hline \multirow[t]{3}{*}{$\Delta F / T q$} & $F \Delta / v 1$ & عصاره كرده كاج •(mg/L)1ه \\
\hline & & P-value \\
\hline & & SEM \\
\hline
\end{tabular}

داراى زنتيك ماده و فنوتيت نر است. وايلانت و همكاران

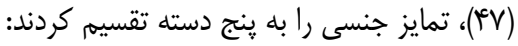

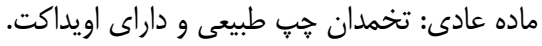

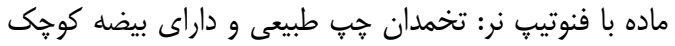

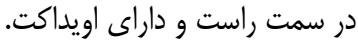

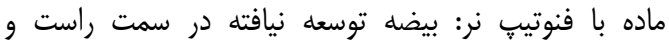

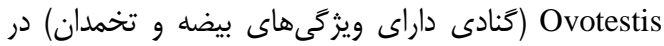

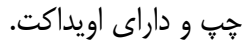

ماده با فنوتيبٍ نر: داراى دو دو بيضه توسعه نيافته، لوله دفرانت نازك و اويداكت.

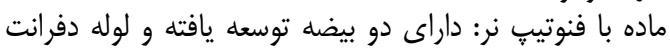
نازى ير از اسِيرم و اويداكت.

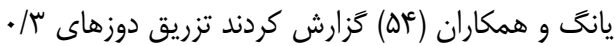

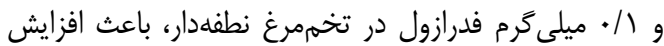

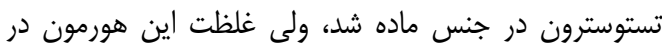

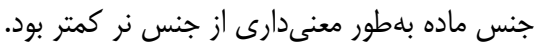

صفات اقتصادى هيج كدام از تيمار آزمايشى تاثير معنى دارى برى بر صفات

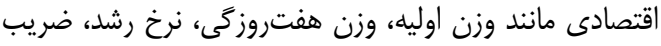

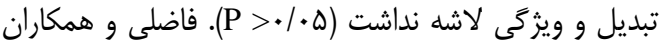

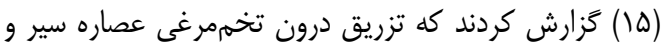

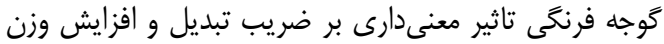

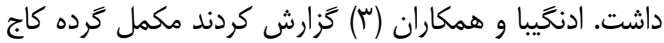

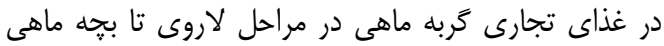

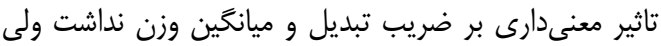

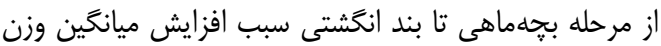

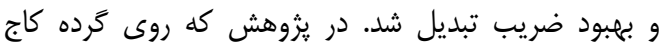

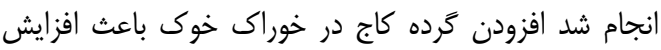

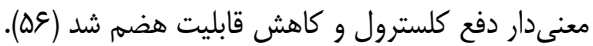

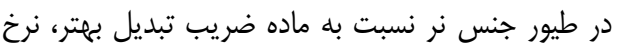

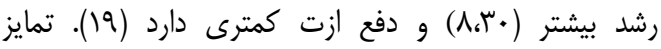

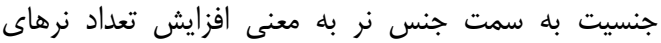


به طور معنى دار كاهش يافت (V)؛ همجنين گزارش شده است

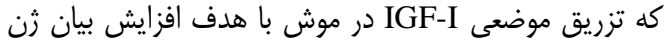
فيرها شد (Y)-I

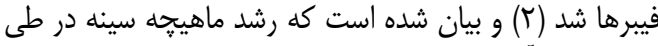

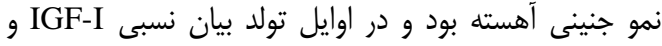

IGF-II

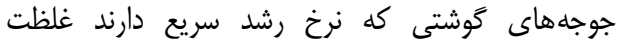

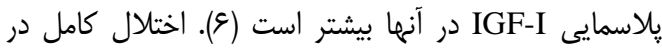

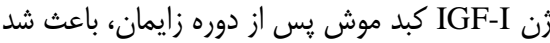

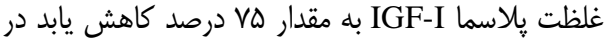

حالى كه نرخ رشد نرمال بود (س山الها

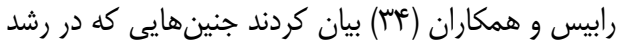

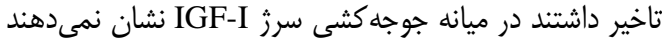

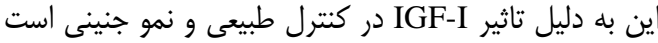
تكثير را از راه افزايش تعداد سلول و سطوح يروتين و ندين و

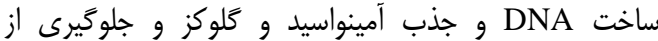

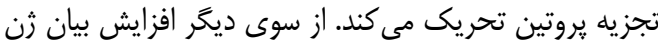

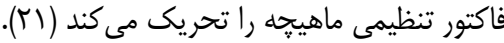

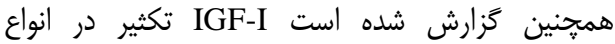

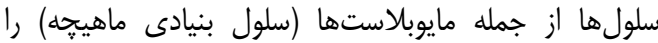

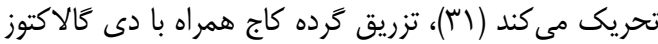

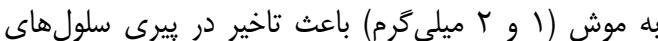

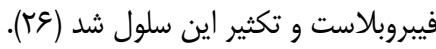

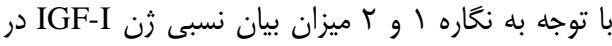

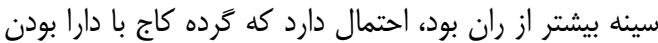

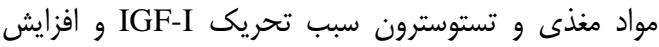

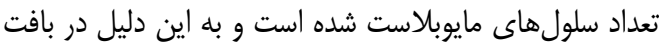
سينه داراى ماهيجه بيشتر نسبت به به ران است است

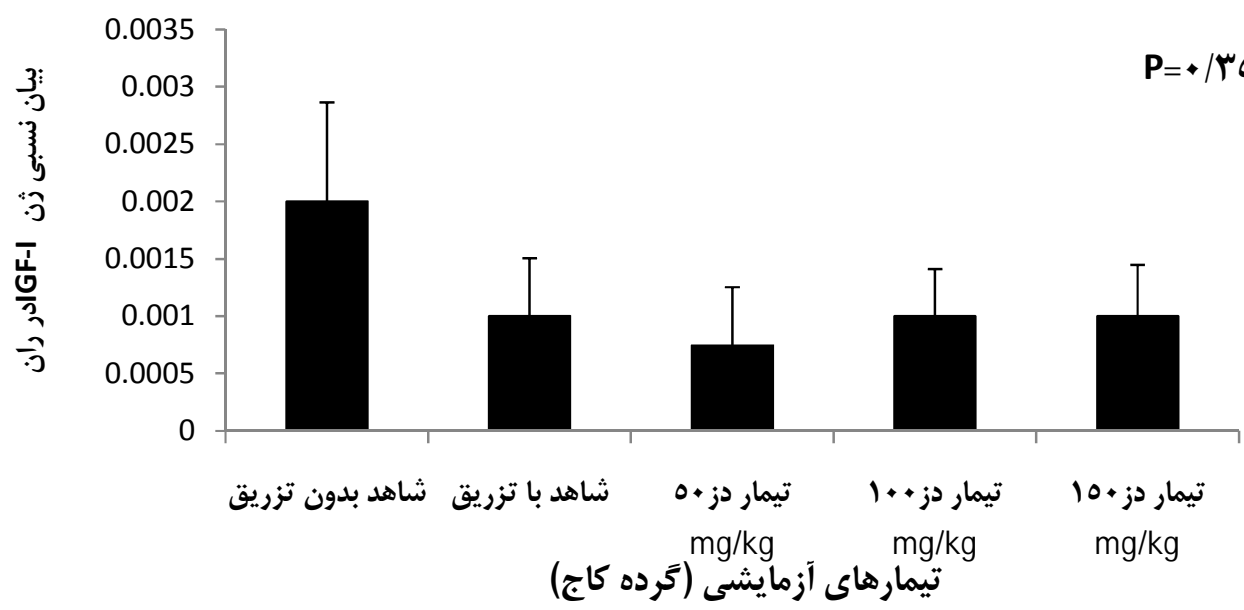

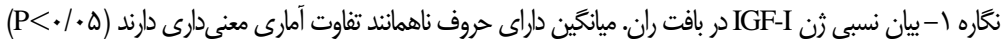

Figure 1. Relative gene expression of IGF-I in thigh tissue. Means bearing different superscript letters differ

Figure 1. Relative gene expression of IGF-I in thigh tissue. Means

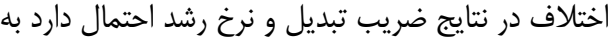

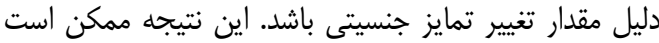

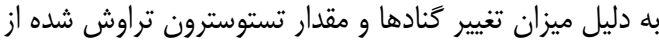
كنادها باشد.

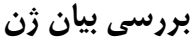

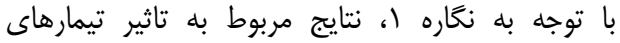

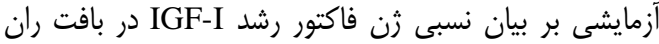

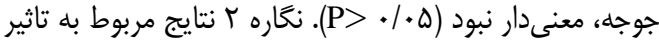
عصاره گرده كاج بر بيان نسبى ثن فاكتور رشد إهد

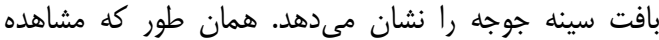

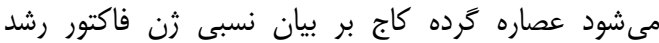
IGF-I در تيمار تزريق شده (شاهد تزريق شده ،تيمار با دز

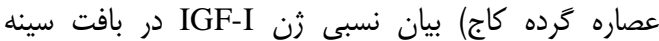

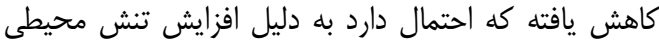

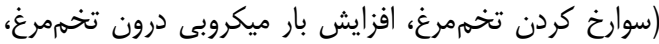

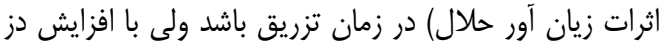

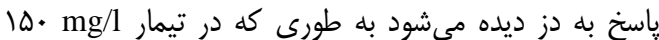

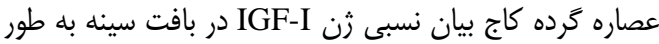

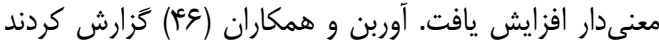

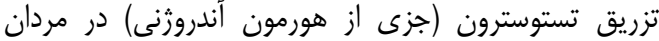

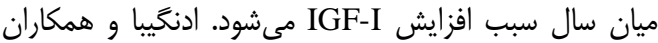

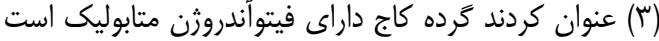

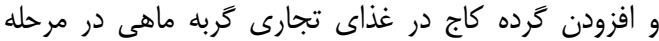

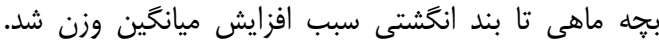

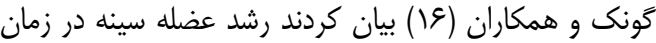

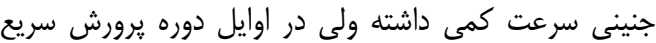

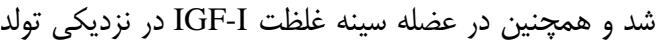

$P=+/$ T०० 


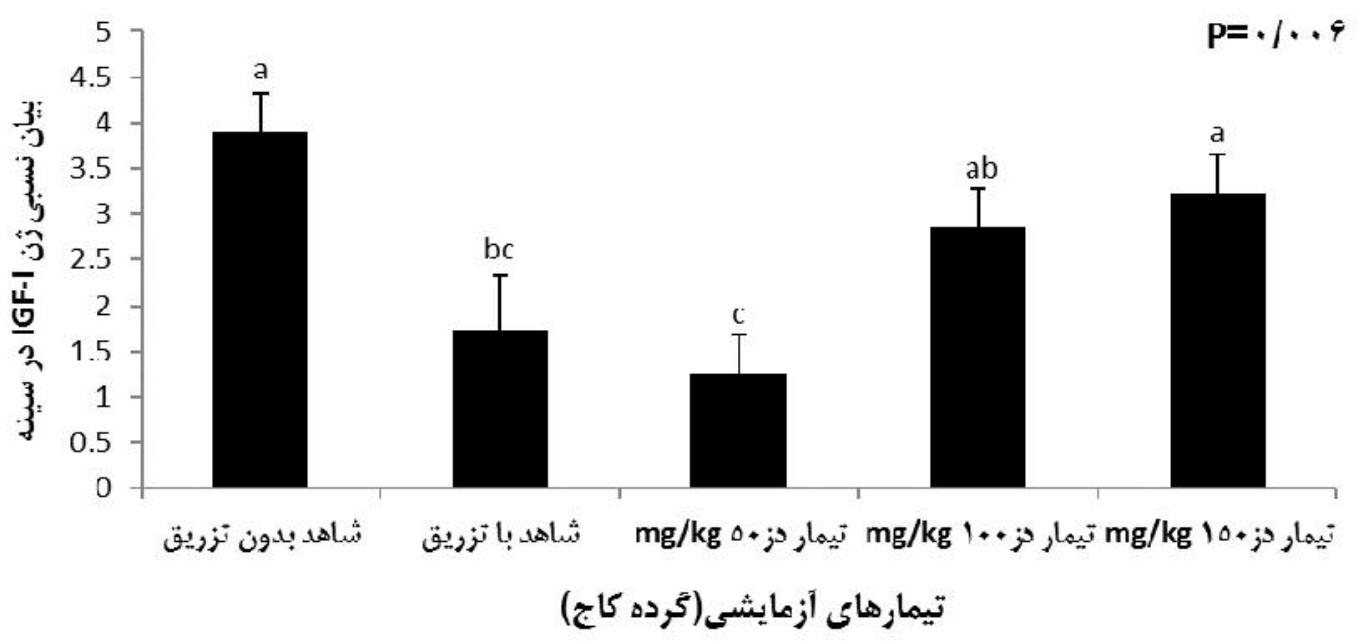

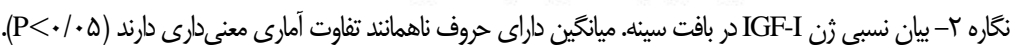

Figure 2. Relative gene expression of IGF-I in breast tissue. Means bearing different superscript letters differ significantly $(\mathrm{P}<0.05)$

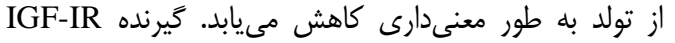

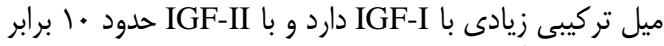

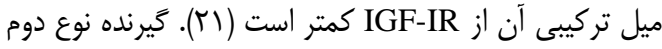

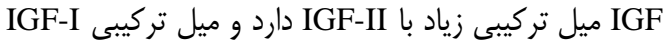

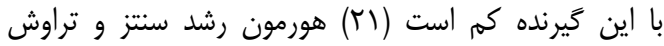

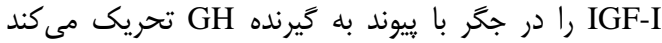

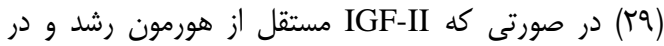

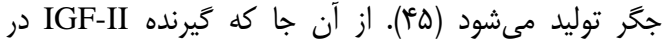

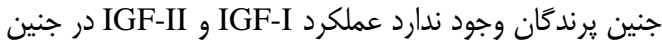

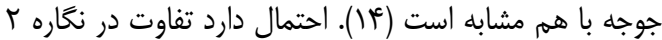

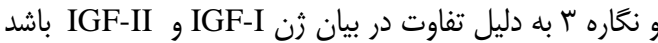

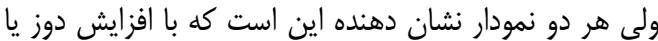

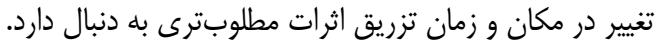

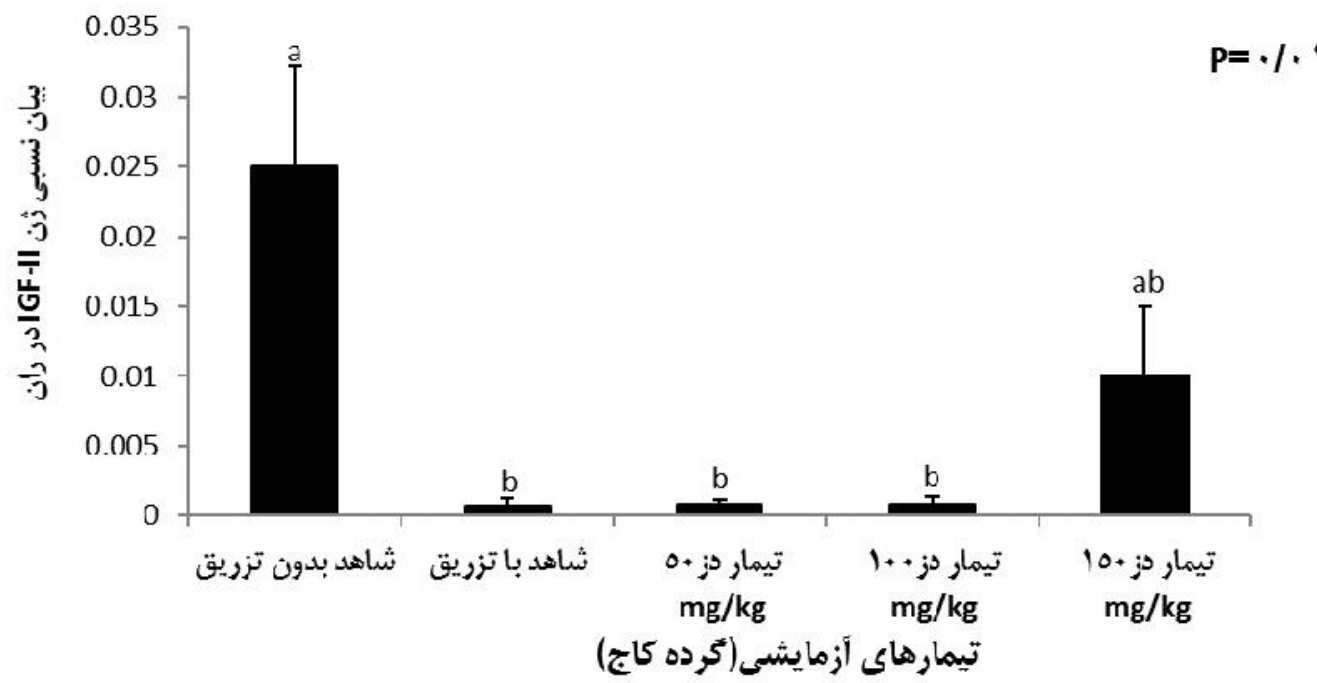

با توجه به نغاره س نتايج مربوط بله تاثير تيمارهاى

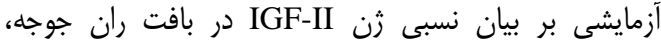

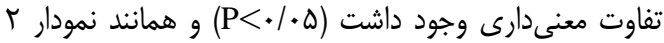

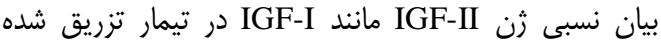

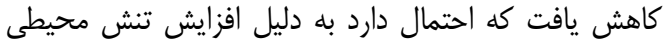

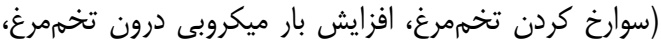

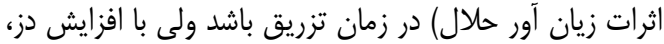

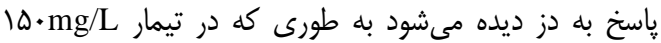

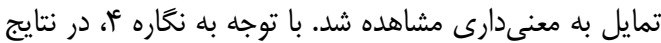
مربوط به تاثير عصاره گرده كاج بر بيان نسبى ثرائ ثن فاكتور رشد IGF-II

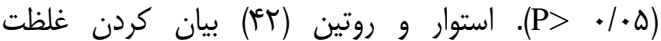

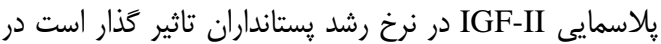
زمان جنينى IGF-II تقريبا بيان نسبى بيشترى دارد، ولى بعد بلى 


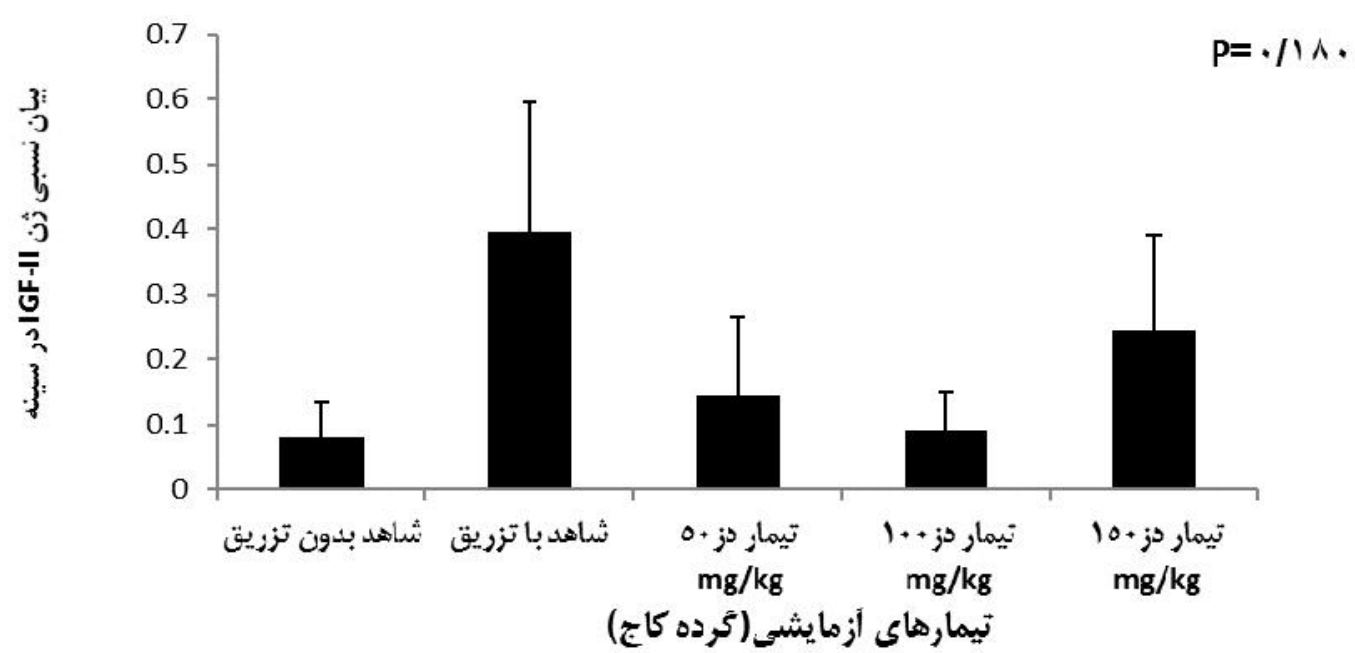

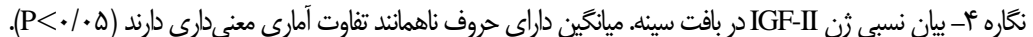

Figure 4. Relative gene expression of IGF-II in breast tissue. Means bearing different superscript letters differ significantly $(\mathrm{P}<0.05)$

$$
\begin{aligned}
& \text { افزايش معنى دار در بيان نسبى IGF-I شد. }
\end{aligned}
$$

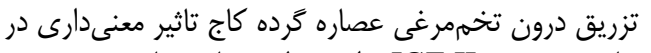

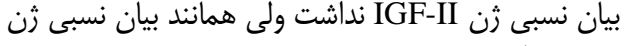

$$
\begin{aligned}
& \text { IGF-I }
\end{aligned}
$$

$$
\begin{aligned}
& \text { تزريق درون تخممرغى عصاره گرده كاج تاثير معنىدارى بر }
\end{aligned}
$$

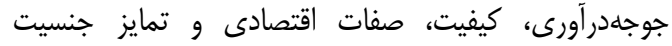

$$
\begin{aligned}
& \text { جوجههاى گوشتى نداشت. }
\end{aligned}
$$

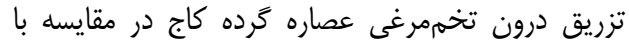

$$
\begin{aligned}
& \text { تيمارهاى با تزريق شده (به خصوص بيشترين سطح آن) سبب }
\end{aligned}
$$

1. Abedi, A., M. Parviz, S.M. Karimian and H.R.S. Rodsari. 2013. Aphrodisiac activity of aqueous extract of Phoenix dactylifera pollen in male rats Journal of Physiolgy and Pharmacology Advances, 2: 235-242.

2. Adams, G.R. and S.A. McCue. 1998. Localized infusion of IGF-I results in skeletal muscle hypertrophy in rats. Journal of Applied Physiology, 84: 1716-1722.

3. Adenigba, I., B. Tumbokon and A. Serrano. 2017. Androgenic and Anabolic effects of Pinus tabulaeformis Carr. Pollen in Clarias gariepinus. The Israeli Journal of Aquaculture, 69: 1388-1396.

4. Allan, G.J., D.J. Flint and K. Patel. 2001. Insulin-like growth factor axis during embryonic development. Reproduction, 122: $31-39$

5. Aslani, M. and M. Mottaghitalab. 2011. Effect anti-aromatase tea extract on the sex ratio of broiler chicks, MS, University of Agricultural Sciences, Gilan, Iran, 80 (In Persian).

6. Bacon, W.L., K.E. Nestor, D.A. Emmerson, R. Vasilatos-Younken and D.W. Long. 1993. Circulating IGF-I in plasma of growing male and female turkeys of medium and heavy weight lines. Domestic Animal Endocrinology, 10: 267-277.

7. Beccavin, C., B. Chevalier, L.A. Cogburn, J. Simon and M.J. Duclos. 2001. Insulin-like growth factors and body growth in chickens divergently selected for high or low growth rate. Journal of Endocrinology, 168: 297-306.

8. Benyi, K., T.S. Tshilate, A.J. Netshipale and K.T. Mahlako. 2015. Effects of genotype and sex on the growth performance and carcass characteristics of broiler chickens. Tropical Animal Health and Production, 47: 1225-1231.

9. Burke, W.H. and M.H. Henry. 1999. Gonadal development and growth of chickens and turkeys hatched from eggs injected with an aromatase inhibitor. Poultry science, 78: 1019-1033.

10. Chu, H.L., H. Mao, W. Feng, J.W. Liu and Y. Geng. 2013. Effects of sulfated polysaccharide from Masson pine (Pinus massoniana) pollen on the proliferation and cell cycle of HepG2 cells. International Journal of Biological Macromolecules, 55: 104-108.

11. Coşkun, I., H. Çayan, O. Yilmaz, A. Taskin, E. Tahtabiçen and H.E. Samli. 2014. Effects of in-ovo pollen extract injection to fertile broiler eggs on hatchability and subsequent chick weight. Turk Tarım ve Doga Bilimleri Dergisi, 1: 485-489.

12. Cobb hatchery management guide. 2001. The cobb Breeding company Ltd. Available on: www.cobbvantress.com

13. Elbrecht, A. and R. Smith. 1992. Aromatase enzyme activity and sex determination in chicken. Science, 255: 464-469.

14. Duclos, M.J., R.S. Wilkie and C. Goddard. 1991. Stimulation of DNA synthesis in chicken muscle satellite cells by insulin and insulin-like growth factors: evidence for exclusive mediation by a type-I insulin-like growth factor receptor. Journal of Endocrinology, 128: 35-NP

15. Fazli, N., A. Hassanabadi, M. Mottaghitalab and H. Hajati. 2015. Manipulation of broiler chickens sex differentiation by in ovo injection of aromatase inhibitors and garlic and tomato extracts. Poultry Science, 94: 2778-2783.

16. Guernec, A., C. Berri, B. Chevalier, N. Wacrenier-Cere, E. Le Bihan-Duval and M.J. Duclos. 2003. Muscle development, insulin-like growth factor-I and myostatin mRNA levels in chickens selected for increased breast muscle yield. Growth Hormone \& IGF Research, 13: 8-18.

17. Hong, I.P., S.O. Woo and J.S. Jang. 2013. Effect on testosterone production in sprague-dawley rats of gastrodia and bee pollen .Journal Environ Health Sciences, 39: 43-47.

18. Hamburger V. and H.L. Hamilton. 1951. A series of normal stages in the development of the chick embryo. Journal of Morphology, 88: 49-92.

19. Hernandez, F., M. Lopez, S. Martinez, M.D. Megias, P. Catala and J. Madrid. 2012. Effect of low-protein diets and single sex on production performance, plasma metabolites, digestibility and nitrogen excretion in 1-to 48-day-old broilers. Poultry Science, 91: 683-692.

20. Kaylashki, A. 2006. Effects of Some Natural Factors on the Quantitative Characteristics of (Pinus eldarica Medw) in the West Forest of Mazandaran. Scientific Journal of Agriculture, 1: 81-87 (In Persian). 
$v^{e}$ تاثير تزريق درون تخممرغى عصاره گرده كاج بر رشد و تمايز جنسيتى جوجه هاى گوشتى 21. Laviola, L., A. Natalicchio and F. Giorgino. 2007. The IGF-I signaling pathway. Current pharmaceutical design, 13:
663-669.

22. Lee, K.H. and E.M. Choi. 2009. Effect of pine pollen extract on experimental chronic arthritis. Phytotherapy Research, 23: 651-657.

23. Liu, Y., W. Guo, Z. Pu, X. Li, X. Lei, J. Yao and X. Yang. 2016. Developmental changes of Insulin-like growth factors in the liver and muscle of chick embryos. Poultry Science, 95(6): 1396-1402.

24. Lu, F.Z., J. Chen, X.X. Wang and H.L. Liu. 2009. Investigation of the insulin-like growth factor system in breast muscle during embryonic and postnatal development in Langshan and Arbor Acres chickens subjected to different feeding regimens. Asian-Australasian Journal of Animal Sciences, 22(4): 471-482

25. Mafia, M., M. Mottaghitalab and M. Hamooni Haghighat. 2010. Manipulation of the Sexual Distribution of Broiler Chickens Using Intraperitoneal Injection of Black Eagle Extract, MS, University of Agricultural Sciences, Guilan, Iran, 79 (In Persian).

26. Mao, G.X., D. Zheng, Y.B. Cao, Z.M. Chen, Y.D. Lv, Y.Z. Wang and J. Yan 2012. Antiaging effect of pine pollen in human diploid fibroblasts and in a mouse model induced by D-galactose. Oxidative medicine and cellular longevity, 85: $112-128$.

27. Mottaghitalab M. and A. Jamshasb. 2016 Effect of in ovo injection of tomato extract on sex differences and economic performance of broiler chickens, Seventh Iranian Congress of Animal Science, Tehran, Iran, (In Persian).

28. Mortensen, A.S. and A. Arukwe. 2006. Dimethyl sulfoxide is a potent modulator of estrogen receptor isoforms and xenoestrogen biomarker responses in primary culture of salmon hepatocytes. Aquatic Toxicology, 79: 99-103.

29. Ohlsson, C., B.A. Bengtsson, O.G. Lsaksson, T.T. Andreassen, and M.C. Slootweg. 1998. Growth hormone and bone. Endocrinol, Rev. 19: 55- 79.

30. Ojedapo, L.O., O. Akinokun, T.A. Adedeji, T.B. Olayeni, S.A. Ameen and S.R. Amao. 2008. Effect of strain and sex on carcass characteristics of three commercial broilers reared in deep litter system in the derived savannah area of Nigeria. World Journal of Agricultural Sciences, 4: 487-491.

31. Quinn, L.S., B. Steinmetz, A. Maas, L. Ong and M. Kaleko. 1994. Type-1 insulin-like growth factor receptor overexpression produces dual effects on myoblast proliferation and differentiation. Journal of cellular physiology, 159: 387-398.

32. Pourabbas, S., M. Kestemi and A.R. Reshk. 2011. Effects anxiolytic of fennel and probabilistic role of gobarergic system and estrogen receptors in these effects in rats. Iranian physiology and pharmacology, Volume 1: 134-143 (In Persian).

33. Rastgar Farajzad, A., K. Ghasemi and R. Idelzadeh. 2013. Ganung Medical Physiology, Ganung. Publishers world of adib $.750 \mathrm{pp}$ (In Persian)

34. Robcis, H.L., T. Caldes and F.D. Pablo. 1991. Insulin-Like Growth Factor-I Serum Levels Show a Midembryogenesis Peak in Chicken That Is Absent in Growth-Retarded Embryos Cultured ex Ovo. Endocrinology, 128: $1895-1901$.

35. Saden-Krehula, M., M. Tajic and D. Kolbah. 1971. Testosterone, epitestosterone and and rostenedione in the pollen of Scotch pine P. silvestris L. Cellular and Molecular Life Sciences, 27: 108-109.

36. Saden-Krehula, M., M. Tajic and D. Kolbah. 1979. Sex hormones and corticosteroids in pollen of Pinus nigra. Phytochemistry, 18: 345-346.

37. Saden-Krehula, M. and M. Tajic. 1987. Vitamin D and its metabolites in the pollen of pine. Part 5: Steroid hormones in the pollen of pine species. Die Pharmazie, 42: 471-472.

38. Schmittgen, T.D. and K.J. Livak 2008. Analyzing real-time PCR data by the comparative CT method. Nature protocols, 3: 1101-1108.

39. Shimada, K. 1998. Gene expression of steroidogenic enzymes in chicken embryonic gonads. Journal of Experimental Zoology Part A: Ecological Genetics and Physiology, 281: 450-460.

40. Shimada, K. and N. Saito. 2000. Molccular mechanisms of sex determination and differentiation Journal of Poultry Science, 37: 3-11

41. Smith, C.A. 2010. Rowley review. Sex determination in birds: A review. Emu, 110: 364-377.

42. Stewart, C.E. and P. Rotwein. 1996. Insulin-like growth factor-II is an autocrine survival factor for differentiating mvoblasts. Journal of Biological Chemistrv. 271: 11330-11338.

43. SAS. 2006. Statistical analysis system proprietary software release 9.1. SAS Inst. Inc.,

44. Tona, K., F. Bamelis, B. De Ketelaere, V. Bruggeman, V.M. Moraes, J. Buyse, O. Onagbesan and Decuypere, E. 2003. Effects of egg storage time on spread of hatch, chick quality, and chick juvenile growth. Poultry Science, 82: $736-741$.

45. Towhidi, A., A. Dirandeh and T. Saberi Fred. 2010. Hormonal regulation of farm animal growth, translated by Hossner, K.L.. first edition Tehran Universitv Press 1-314. .( In Persian)

46. Urban. R.J.. Y.H. Bodenburg. C.H.A.R.L.E. S. Gilkison. Y.J.U.D. Foxworth. A.R. Coggan. R.R. Wolfe and A Ferrando.. 1995. Testosterone administration to elderlv men increases skeletal muscle strength and protein synthesis. American Journal of Physiology-Endocrinology and Metabolism, 269: 820-826.

47. Vaillant, S., D. Guemene, M. Dorizzi, C. Pieau, N. Richard-Mercier and J.P. Brillard. 2003. Degree of sex reversal as related to plasma steroid levels in genetic female chickens (Gallus domesticus) treated with Fadrozole. Molecular Reproduction and Development, 65: 420-428.

48. Valizadeh, E. and H. Seratinouri. 2013. Effects of garlic extract, anti-estrogens, and aromatase inhibitor on sex differentiation in embryo. International Journal of Women's Health and Reproduction Sciences, 1: 51-55.

49. Weissmann, A., S. Reitemeier, A. Hahn, J. Gottschalk and A. Einspanier. 2013. Sexing domestic chicken before hatch: A new method for in ovo gender identification. Theriogenology, 80: 199-205.

50. Willemsen, H., N. Everaert, A. Witters, L. De Smit, M. Debonne, F. Verschuere, P. Garain, D. Berckmans, E Decuypere and V. Bruggeman. 2008. Critical assessment of chick quality measurements as an indicator of posthatch performance. Poultry Science, 87: 2358-2366.

51. Willemsen, H., B. Kamers, F. Dahlke, H. Han, Z. Song, Z. Ansari Pirsaraei, T. Kokou, D. Eddy and N. Everaert. 2010. High-and low-temperature manipulation during late incubation: effects on embryonic development, the hatching process, and metabolism in broilers. Poultry Science, 89: 2678-2690.

52. Wyatt, R.D. and B. Howarth. 1976. Effect of dimethyl sulfoxide on embryonic survival and subsequent chick nerformance. Poultrv Science. 55: 579-582

53. Yakar. S.J.. L. Liu. B. Stannard. A. Butler. D. Accili. B. Sauer and D. LeRoith. 1999. Normal growth and develonment in the absence of hepatic insulin-like growth factor I. Proceedings of the National Academy of Sciences, 96: 7324-7329.

54. Yang, X.R., H.S. Jiang, J.X. Zheng, L.J. QU, S.R. Chen, J.Y. LI and Y.A.N.G. Ning. 2013. Dosage Effects of Fadrozole on Growth and Development of Sex-Reversed Genetic Female Chickens. Journal of Integrative Agriculture, 12: 10491053 .

55. Zamiri M.G. 2001 Reproduction in domestic birds, translated by Robert, J. Charles Part IV: 134-95 (In Persian)

56. Zhao, L., W. Windisch and M. Kirchgessner. 1996. A study on the nutritive value of pollen from the Chinese Masson Pine (Pinus massoniana) and its effect on fecal characteristics in rats. Zeitschrift fur Ernahrungswissenschaft, 35: 341347. 


\title{
Effect of in ovo Injection of Pine Pollen Extract on Growth and Sex Differences of Broiler Chicks
}

\section{Afsane Sarbozi Farah Abad ${ }^{1}$ Zarbakht Ansari-Pirsaraei $^{2}$ Pourya Biparva $^{3}$ and} Essa Dirandeh $^{4}$

1, 3 and 4- Graduated M.Sc. Student of Animal Sciences Department, Assistant Professor of Basic Sciences Department and Assistant Professor of Animal Sciences Department, Sari Agricultural Sciences and Natural

Resources University

2- Associate Professor of Animal Sciences Department, Sari Agricultural Sciences and Natural Resources University, (Corresponding Author:zarbakht_ansari@yahoo.com)

\author{
Received: August 20,2017 Accepted: January 10, 2018
}

\begin{abstract}
One of the problems of the poultry industry is a lack of flocks in broiler farms Although it in the factories of hatchery broiler chickens and turkey, often Sexing chickens to are selling commercial growers, because sex differences in growth rate and feed efficiency, separating males and females to the necessary recurrence needs of uniform and specified market in terms of production carcasses. The current study was conducted to evaluate the effect of in ovo injection of Pine pollen extract on growth and sex differentiation of broiler chicks. A total of 500 Ross (W-308) egg completely randomized with five treatments were assigned to 4 replicate of 25 eggs in each pen. Treatments included: 1-.Non-injected control 2-Sham control (DMSO) 3- injection $50 \mathrm{mg} / \mathrm{L}$ of Pine pollen extract 4-injection $100 \mathrm{mg} / \mathrm{L}$ of Pine pollen extract 5injection $150 \mathrm{mg} / \mathrm{L}$ of Pine pollen extract. The economic characteristics, hatching percent and the sex differentiation and the relative expression of IGF-I and IGF-II were evaluated. The results of this study showed that Pine pollen extract had no significant effect on hatching percent, economic characteristics and the sex differentiation; but, in ovo injection of Pine pollen extract decreased the relative expression of IGF-I and IGF-II genes. Relative expression of IGFI gene in breast tissue and IGF-II in the thigh tissue had a dose response to Pine pollen extract. Injection of $150 \mathrm{mg} / \mathrm{L}$ of pine pollen extract significantly increased relative gene expression of IGF-I in breast tissue.
\end{abstract}

Keywords: In ovo injection, Sex differentiation, Growth, Pine pollen extract 